\title{
Large deformation finite element analysis of micropipette aspiration to determine the mechanical properties of the chondrocyte
}

Citation for published version (APA):

Baaijens, F. P. T., Trickey, W. R., Laursen, T. A., \& Guilak, F. (2005). Large deformation finite element analysis of micropipette aspiration to determine the mechanical properties of the chondrocyte. Annals of Biomedical Engineering, 33(4), 494-501. https://doi.org/10.1007/s10439-005-2506-3

DOI:

10.1007/s10439-005-2506-3

Document status and date:

Published: 01/01/2005

Document Version:

Publisher's PDF, also known as Version of Record (includes final page, issue and volume numbers)

Please check the document version of this publication:

- A submitted manuscript is the version of the article upon submission and before peer-review. There can be important differences between the submitted version and the official published version of record. People interested in the research are advised to contact the author for the final version of the publication, or visit the $\mathrm{DOI}$ to the publisher's website.

- The final author version and the galley proof are versions of the publication after peer review.

- The final published version features the final layout of the paper including the volume, issue and page numbers.

Link to publication

\footnotetext{
General rights

- You may freely distribute the URL identifying the publication in the public portal. follow below link for the End User Agreement:

www.tue.nl/taverne

Take down policy

If you believe that this document breaches copyright please contact us at:

openaccess@tue.nl

providing details and we will investigate your claim.
}

Copyright and moral rights for the publications made accessible in the public portal are retained by the authors and/or other copyright owners and it is a condition of accessing publications that users recognise and abide by the legal requirements associated with these rights.

- Users may download and print one copy of any publication from the public portal for the purpose of private study or research.

- You may not further distribute the material or use it for any profit-making activity or commercial gain

If the publication is distributed under the terms of Article 25fa of the Dutch Copyright Act, indicated by the "Taverne" license above, please 


\title{
Large Deformation Finite Element Analysis of Micropipette Aspiration to Determine the Mechanical Properties of the Chondrocyte
}

\author{
FrANK P. T. BAAIJENS, ${ }^{1}$ WENDY R. TRICKEy,${ }^{2}$ TOD A. LAURSEN,${ }^{3}$ and FARSHID GUILAK ${ }^{2}$ \\ ${ }^{1}$ Department of Biomedical Engineering, Eindhoven University of Technology, Eindhoven, The Netherlands; ${ }^{2}$ Departments of Surgery \\ and Biomedical Engineering, Duke University Medical Center, Durham, NC; and ${ }^{3}$ Department of Civil \\ and Environmental Engineering, Duke University, Durham, NC
}

(Received 24 May 2004; accepted 8 November 2004)

\begin{abstract}
Chondrocytes, the cells in articular cartilage, exhibit solid-like viscoelastic behavior in response to mechanical stress. In modeling the creep response of these cells during micropipette aspiration, previous studies have attributed the viscoelastic behavior of chondrocytes to either intrinsic viscoelasticity of the cytoplasm or to biphasic effects arising from fluid-solid interactions within the cell. However, the mechanisms responsible for the viscoelastic behavior of chondrocytes are not fully understood and may involve one or both of these phenomena. In this study, the micropipette aspiration experiment was modeled using a large strain finite element simulation that incorporated contact boundary conditions. The cell was modeled using finite strain incompressible and compressible elastic models, a twomode compressible viscoelastic model, or a biphasic elastic or viscoelastic model. Comparison of the model to the experimentally measured response of chondrocytes to a step increase in aspiration pressure showed that a two-mode compressible viscoelastic formulation accurately captured the creep response of chondrocytes during micropipette aspiration. Similarly, a biphasic two-mode viscoelastic analysis could predict all aspects of the cell's creep response to a step aspiration. In contrast, a biphasic elastic formulation was not capable of predicting the complete creep response, suggesting that the creep response of the chondrocytes under micropipette aspiration is predominantly due to intrinsic viscoelastic phenomena and is not due to the biphasic behavior.
\end{abstract}

Keywords-Cartilage, Osteoarthritis, Cytoskeleton, Biomechanics, Poroelastic, Poroviscoelastic, Nonlinear.

\section{INTRODUCTION}

Chondrocytes are responsible for the maintenance of articular cartilage through the anabolism and catabolism of the extracellular matrix components. With osteoarthritis $(\mathrm{OA})$, this homeostasis is altered ${ }^{15}$ leading to progressive degeneration of the tissue. The chondrocyte's response to biophysical factors engendered in the joint, such as stresses,

Address correspondence to Frank P. T. Baaijens,Department of Biomedical Engineering, Eindhoven University of Technology, Eindhoven, The Netherlands. Electronic mail: f.p.t.baaijens@tue.nl strains, and fluid flow, is believed to contribute in part to the maintenance of the cartilage extracellular matrix. ${ }^{6}$ Thus, a characterization of the mechanical environment of the chondrocyte and how this environment is altered with OA would provide important insight on the regulation of chondrocyte physiology in health and disease.

To gain a better understanding of the mechanical environment at a cellular level, mathematical models have been developed of the chondrocyte within its extracellular matrix while under compression, where the cell and matrix are modeled as biphasic materials. ${ }^{2,25}$ In order to predict accurately the spatial distribution and time history of the biophysical factors within and around the chondrocyte, such models require knowledge of the constitutive material behavior and mechanical properties of the cells and the extracellular matrix.

The micropipette aspiration technique has been used extensively in previous studies to determine the mechanical properties of cells (Fig. 1). ${ }^{9}$ While most such studies have focused on circulating cells, which behave as a liquid drop with cortical tension, certain cell types such as endothelial cells, osteoblasts, and chondrocytes behave as viscoelastic solid materials. ${ }^{11,16,19,20,22}$

Previous studies have also used the micropipette technique to determine the volume change of chondrocytes after complete aspiration into a micropipette. ${ }^{11}$ These studies demonstrated that chondrocytes are not incompressible, in that significant volume decreases were observed with compression. In other studies ${ }^{23}$ these experiments were used to show that the Poisson's ratio of the chondrocyte is approximately 0.4 , providing a material property of the cell which is independent of the specific experimental configuration.

However, in modeling the mechanical behavior of these cells, previous studies have attributed the viscoelastic behavior of chondrocytes to either intrinsic viscoelasticity of the cytoplasm ${ }^{7,12,22}$ or to biphasic effects arising from fluid-solid interactions within the cell. ${ }^{2,5}$ However, the mechanisms responsible for the viscoelastic behavior of 

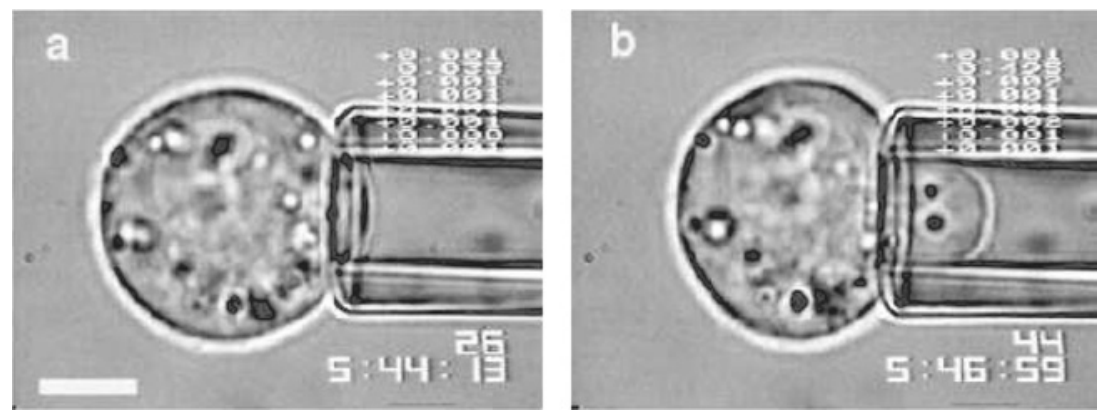

FIGURE 1. Micropipette aspiration of a chondrocyte. (a) Before and (b) after partial aspiration of a cell into a micropipette for the determination of the Young's modulus. Scale bar $=5 \mu \mathrm{m}$. The applied pressure equals $500 \mathrm{~Pa}$. The ratio of the diameter of the cell and the micropipette $D_{\mathrm{c}} / D_{\mathrm{p}}=2.65$.

chondrocytes are not fully understood, and may involve one or both of these phenomena.

The goal of this study was to examine the hypothesis that the transient behavior of the chondrocyte in the micropipette aspiration experiment derives from solid-solid interactions (intrinsic viscoelasticity), fluid-solid interactions (biphasic behavior), or both (biphasic poroviscoelasticity). ${ }^{18}$ To simulate the steady state aspiration length during micropipette aspiration, the cell was modeled using finite strain incompressible and compressible elastic models. The creep response was investigated using a finite strain two-mode compressible viscoelastic model, a biphasic elastic model, and a biphasic two-mode viscoelastic model. The cell was modeled as a nonlinear homogeneous compressible elastic sphere in the finite element model, as opposed to previous studies that have assumed a linear homogeneous incompressible elastic half-space in the analytical solution. ${ }^{7,12,16,20}$ In this manner, a secondary goal was to examine the influence of the geometry of the cell and micropipette on the measured cell properties, in comparison to analytical solutions of micropipette aspiration.

\section{METHODS}

A nonlinear finite element analysis of the micropipette experiment was performed using several constitutive models. Neglecting the effect of inertia terms and body forces, the governing momentum equation is expressed as

$$
\vec{\nabla} \cdot \sigma=\overrightarrow{0}
$$

and must be supplemented by suitable boundary conditions.

To study the elastic properties of the cell a compressible Neo-Hookean model was adopted, expressed as

$$
\boldsymbol{\sigma}=\kappa(J-1) \boldsymbol{I}+\frac{G}{J}\left(\boldsymbol{B}-J^{2 / 3} \boldsymbol{I}\right)
$$

where, using the deformation tensor $\boldsymbol{F}=\left(\vec{\nabla}_{0} \vec{x}\right)^{T}$ with $\vec{\nabla}_{0}$ the gradient operator with respect to the reference configuration, the volume ratio $J$ is defined as

$$
J=\operatorname{det}(\boldsymbol{F}),
$$

and the right Cauchy-Green tensor $\boldsymbol{B}$ is given by

$$
\boldsymbol{B}=\boldsymbol{F} \cdot \boldsymbol{F}^{T} .
$$

The material parameters $\kappa$ and $G$ denote the compressibility modulus and the shear modulus, respectively. These parameters may be expressed in terms of the Young's modulus and the Poisson's ratio, via

$$
G=\frac{E}{2(1+v)}, \quad \kappa=\frac{E}{3(1-2 v)}
$$

In the limit of incompressible behavior $J=\operatorname{det}(\boldsymbol{F})=1$ or, equivalently, $v=0.5$, and this model reduces to

$$
\boldsymbol{\sigma}=-p \boldsymbol{I}+\boldsymbol{\tau},
$$

with

$$
\boldsymbol{\tau}=G(\boldsymbol{B}-\boldsymbol{I}) .
$$

A viscoelastic model is used to investigate the timedependent behavior of the cell during micropipette aspiration. For this purpose, a two-mode viscoelastic model is introduced

$$
\sigma=\sigma_{\mathrm{e}}+\boldsymbol{\tau}_{\mathrm{v}}
$$

where $\sigma_{\mathrm{e}}$ denotes a purely elastic response, using Eq. (2) while $\boldsymbol{\tau}_{\mathrm{v}}$ denotes a viscoelastic response, employing a compressible Upper Convected Maxwell model

$$
\stackrel{\nabla}{\boldsymbol{v}}_{\mathrm{v}}+\frac{1}{\lambda} \boldsymbol{\tau}_{\mathrm{v}}=2 G_{\mathrm{v}} \boldsymbol{D}^{d}
$$

with $\boldsymbol{D}^{d}$ the deviatoric part of the rate of deformation tensor, defined by

$$
\boldsymbol{D}=\frac{1}{2}\left(\dot{\boldsymbol{F}} \cdot \boldsymbol{F}^{-1}+\boldsymbol{F}^{-T} \cdot \dot{\boldsymbol{F}}^{T}\right)
$$

and $G_{\mathrm{v}}$ the modulus and $\lambda$ the relaxation time of the viscoelastic mode, respectively. The finite element implementation is based on the incrementally objective algorithm. ${ }^{1}$

A biphasic theory is employed to investigate if the time-dependent behavior of the cell during micropipette aspiration can be captured with this theory. Assuming 
isotropic and constant permeability, the governing equations may be cast into ${ }^{13,17}$

$$
\begin{gathered}
\vec{\nabla} \cdot \sigma-\vec{\nabla} p=\overrightarrow{0} \\
\vec{\nabla} \cdot \vec{v}-\vec{\nabla} \cdot k \vec{\nabla} p=0
\end{gathered}
$$

where $\sigma$ denotes the stress tensor of the solid phase, $\vec{v}$ the velocity of the solid phase, $k$ the permeability coefficient, and $p$ the pressure. The fluid-solid interaction is assumed to obey Darcy's law, hence

$$
\vec{v}^{f}-\vec{v}=-k \vec{\nabla} p .
$$

where $\vec{v}^{f}$ denotes the fluid velocity. The stress tensor for the solid phase may either be described by the compressible Neo-Hookean model Eq. (2) or the two-mode viscoelastic model Eqs. (8)-(9). In the actual implementation use is made of the equality $\dot{J} / J=\vec{\nabla} \cdot \vec{v}$, such that Eq. (12) can be written as

$$
\frac{\dot{J}}{J}-\vec{\nabla} \cdot k \vec{\nabla} p=0
$$

During the micropipette aspiration experiments, the cell is exposed to a fluid pressure, $\Delta p$, within the micropipette. The surface traction on the part of the boundary of the cell that is within the micropipette is therefore found from the hydrostatic pressure and the surface normal to the cell. As the cell is aspirated into the micropipette with increasing pressure, the surface normal vectors change with the evolving cell shape. To retain quadratic convergence of the Newton-Raphson iteration process, it proved crucial to include the effect of the rotating normal appearing in the surface traction expression (both due to the external traction and contact pressure) in the stiffness matrix.

The micropipette aspiration experiment was analyzed using an axisymmetric finite element mesh that was refined near the surface contacting the pipette (Fig. 2). To satisfy the so-called inf-sup condition, the elements employ a biquadratic interpolation for the displacement and a bilinear, continuous interpolation for the pressure. Along the left boundary symmetry conditions are applied, while along the portion of the cell boundary within the micropipette suction pressure is applied. Sliding contact conditions along the interface with the micropipette are enforced through the use of a Lagrange multiplier formulation. Mesh refinement studies have indicated that this mesh is sufficiently refined to provide accurate results.

\section{RESULTS}

In response to a step increase in pressure through a micropipette, the chondrocyte demonstrated an initial elastic response to the application of a pressure difference $\Delta p$,

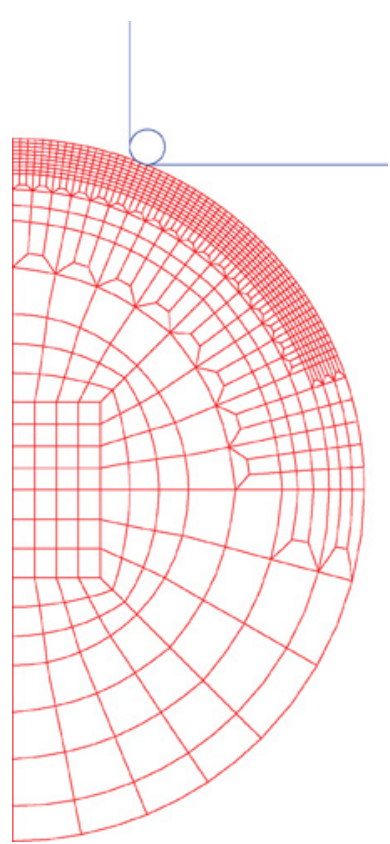

FIGURE 2. Finite element mesh of the micropipette aspiration simulation. The mesh contains 906 elements and has 3243 nodes. To satisfy the so-called inf-sup condition, the elements employ a biquadratic interpolation for the displacement and a bilinear, continuous interpolation for the pressure. Along the left boundary symmetry conditions are applied, while along the portion of the cell boundary within the micropipette suction pressure is applied. Sliding contact conditions along the interface with the micropipette are enforced through the use of a Lagrange multiplier formulation.

followed by a time dependent creep response. The experiments reported previously by Trickey et al. ${ }^{22}$ were fitted with a viscoelastic model formulated Sato et al. ${ }^{16}$ based on a half space approximation assuming incompressible behavior and infinitesimal strains, extending the elastic model of Theret $e t a l .{ }^{20}$ In this model the normalized aspiration length is given by

$$
\frac{a}{R_{\mathrm{p}}}=\frac{\Phi \Delta p}{\pi k_{1}}\left(1-\frac{k_{2}}{k_{1}+k_{2}} e^{-t / \tau}\right)
$$

where $t$ is the time, $\Delta p$ the applied pressure, $a$ the aspiration length, $R_{\mathrm{p}}$ the inner radius of the micropipette, $\Phi$ a wall function identified as 2.1 in the experiments of Trickey et al., ${ }^{22}$ and $k_{1}$ and $k_{2}$ are viscoelastic parameters. The parameters $k_{1}, k_{2}$, and $\tau$ are fitted to experimental data ${ }^{22}$ and are summarized in Table 1. For the experimental protocol (published previously), chondrocytes were isolated from adult human articular cartilage using enzymatic digestion. Cells were placed in a testing chamber on an inverted microscope, ${ }^{9}$ and a tare pressure of $0.01 \mathrm{kPa}$ was applied for $60 \mathrm{~s}$ to define a reference state for measurements of cell deformation. The aspiration experiment involved application of a step increase in pressure ranging from 0.14 to 0.70 $\mathrm{kPa}$, and the length of cell projection into the micropipette was measured for $180 \mathrm{~s}$. The micropipette diameter equals $5 \mu \mathrm{m}$. 
TABLE 1. $k_{1}, k_{2}$ and $\tau$ are parameters of the viscoelastic model according to Sato et al. ${ }^{16}$ and have been identified by Trickey et al. ${ }^{22}$ for chondrocytes of non-osteoarthritic cartilage.

\begin{tabular}{lc}
\hline$k_{1}(\mathrm{kPa})$ & $0.24 \pm 0.11$ \\
$k_{2}(\mathrm{kPa})$ & $0.16 \pm 0.08$ \\
$\tau(\mathrm{s})$ & $33 \pm 20$ \\
$D_{\mathrm{c}}(\mu \mathrm{m})$ & $13.7 \pm 1.5$ \\
\hline
\end{tabular}

Note. $D_{\mathrm{c}}$ is the diameter of the cell.

\section{Equilibrium Response}

The final aspiration length corresponds to an elastic state. In fact, release of the chondrocyte from the micropipette results in a complete recovery of the original spherical shape of the chondrocyte. For $t \rightarrow \infty$ the time dependent contribution, Eq. (9), to the viscoelastic constitutive model, combining Eqs. (2), (8), and (9), vanishes and the model reduces to a purely elastic model, Eq. (2). Therefore, if the Poisson's ratio is known computation of the equilibrium state as a function of the aspiration pressure allows identification of the Young's modulus $E$ by comparison with the steady state response of Eq. (15). In a separate study, ${ }^{21}$ we demonstrate that, on average, chondrocytes have a Poisson's ratio of $v=0.4$.

At three cell/micropipette diameter ratios $D_{\mathrm{c}} / D_{\mathrm{p}}$, we have computed the normalized aspiration length $a / R_{\mathrm{p}}$ as a function of the normalized suction pressure $\Delta p / E$ for a compressible chondrocyte at $v=0.4$, denoted by the closed symbols in Fig. 3. The cell/micropipette diameter ratio has a marked effect on the aspiration length, in particular for small $D_{\mathrm{c}} / D_{\mathrm{p}}$ ratios.

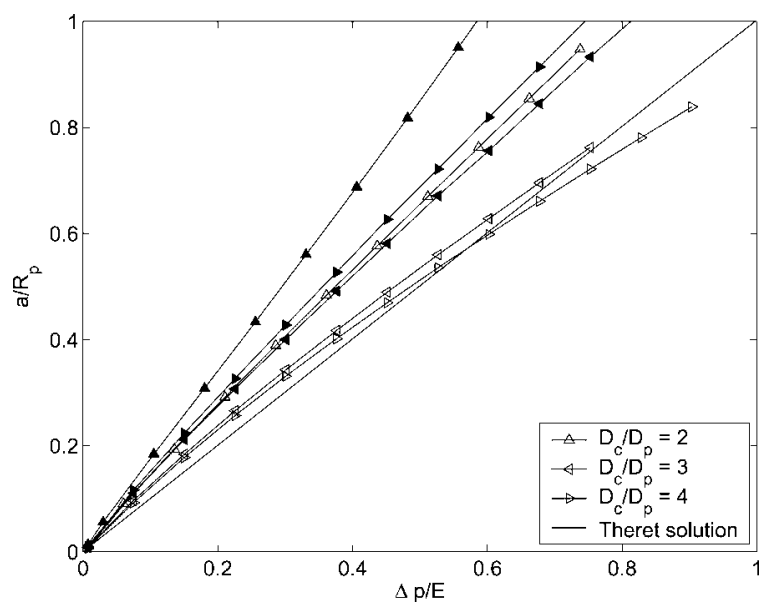

FIGURE 3. Normalized aspiration length $a / R_{\mathrm{p}}$ as a function of the normalized pressure difference $\Delta p / E$. The lines with the open symbols correspond to the incompressible elastic model of the chondrocyte, while the lines with the closed symbols correspond to the compressible elastic model of the chondrocyte, assuming a Poisson's ratio $v=0.4$.
These results may be compared with the experimental fit based on Eq. (15). For $t \rightarrow \infty$ Eq. (15) and $E=3 / 2 k_{1}$ provide a simple expression for the normalized aspiration length that reduces to the elastic model of Theret $e t$ al. ${ }^{20}$

$$
\frac{a}{R_{\mathrm{p}}}=\frac{3 \Delta p \Phi}{2 E \pi}
$$

This expression has been used in various studies to extract the equilibrium modulus $E$ from micropipette experiments. ${ }^{11,22}$ Although Eq. (16) fits the experimental observations fairly accurately, ${ }^{22}$ it may be questioned if the resulting modulus $E$ is meaningful because the model is based on a half space approximation at infinitesimal strains assuming incompressible behavior. In view of the finite size of the chondrocyte relative to the micropipette diameter the use of this expression may be questioned. To investigate the effect of these approximations, the analytical solution was compared to the incompressible, elastic finite element solution at various cell/micropipette diameter ratios represented by the open symbols in Fig. 3. In contrast with the analytical approximation, the finite element simulation predicts a slightly convex response of the aspiration length as a function of the pressure. For a sufficiently large $D_{\mathrm{c}} / D_{\mathrm{p}}$ ratio, hence for sufficiently small micropipette diameters relative to the chondrocyte, results of the computational model were found to be in good agreement with the halfspace theory. ${ }^{20}$ However, it is important to note that, at least locally, the deformations and rotations were found to be large, and exceed the limit of the infinitesimal strain theory. Thus, although the infinitesimal strain theory predicted the aspiration length fairly well for a number of situations, the local deformations near the contact surface are not predicted well at all.

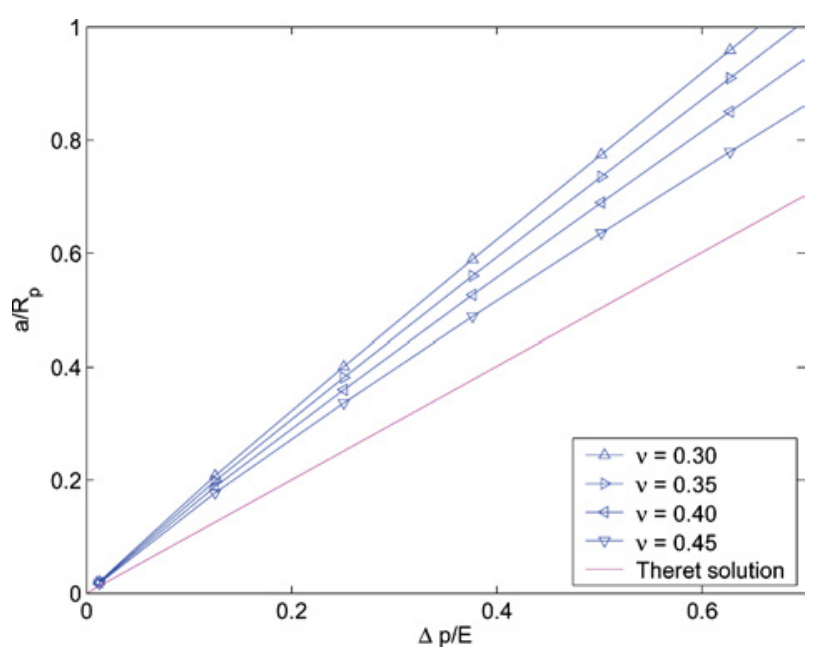

FIGURE 4. Normalized aspiration length $a / R_{p}$ as a function of the normalized pressure difference $\Delta p / E$, assuming a compressible neo-Hookean material model. 
TABLE 2. Slope $c$ of the $\frac{a}{R_{\mathrm{p}}}=c \frac{\Delta p}{E}$ curve.

\begin{tabular}{lllll}
\hline$v$ & 0.45 & 0.4 & 0.35 & 0.3 \\
$c$ & 1.21 & 1.33 & 1.43 & 1.51 \\
\hline
\end{tabular}

Note. This linear expression is used to fit the curves of Fig. 4.

The full aspiration experiments of Jones et al. ${ }^{11}$ indicate that chondrocytes are compressible with a Poisson's ratio of approximately $0.4 .^{23}$ The impact of compressibility on the aspiration length was investigated by adopting the compressible neo-Hookean model Eq. (2) and by varying the Poisson's ratio $v$ while maintaining a constant Young's modulus $E$ (Fig. 4). With increasing compressibility (i.e., decreasing $v)$, the deviation from the incompressible half solution increases. The normalized aspiration length $\left(a / R_{\mathrm{p}}\right)$ is a slightly convex function of the normalized pressure $(\Delta p / E)$, but it may be approximated with a linear function. The slope of this linear approximation as a function of the Poisson ratio is given in Table 2, and is indicative of the deviation from the incompressible half space solution. For example, at $v=0.3$ the slope of the compressible solution deviates $50 \%$ from the slope of incompressible half space solution.

The steady aspiration length was also analyzed using the compressible elastic two-phase model. In that case the Cauchy stress tensor in Eq. (11) is assumed to be the compressible neo-Hookean expression Eq. (2). As before the Poisson's ratio was chosen as $v=0.4$. The hydraulic permeability parameter $k$ in Eq. (12) was estimated at $7.7 \times 10^{-15} \mathrm{~m}^{4} /(\mathrm{N} \cdot \mathrm{s}),{ }^{22}$ which is a suitable upper limit for the permeability parameter. ${ }^{21}$ The results of the analysis are sensitive to the prescribed pressure boundary conditions. At the free surface of the chondrocyte within the pipette, the aspiration pressure was prescribed. At the part of the cell boundary that is in contact with the pipette, the pressure gradient was set to zero, corresponding to a zero fluid velocity. At the part of the boundary outside the pipette, the pressure was set to zero. By performing a series of time dependent simulations at various aspiration pressures $\Delta p$ the steady aspiration length was obtained, and is compared to the compressible and incompressible elastic results as well as the Theret solution (Fig. 5), all for $D_{\mathrm{c}} / D_{\mathrm{p}}=3$. The steady state aspiration length predicted by the elastic two-phase theory is in between the predictions of the compressible and incompressible elastic theory.

\section{Time Dependent Response}

The time-dependent response was analyzed using either the two-mode viscoelastic model or the biphasic theory. Based on previous experiments, ${ }^{11,23}$ a response for an "average" chondrocyte may be defined, which is depicted by the symbols in Fig. 6. In case of the two-mode viscoelastic

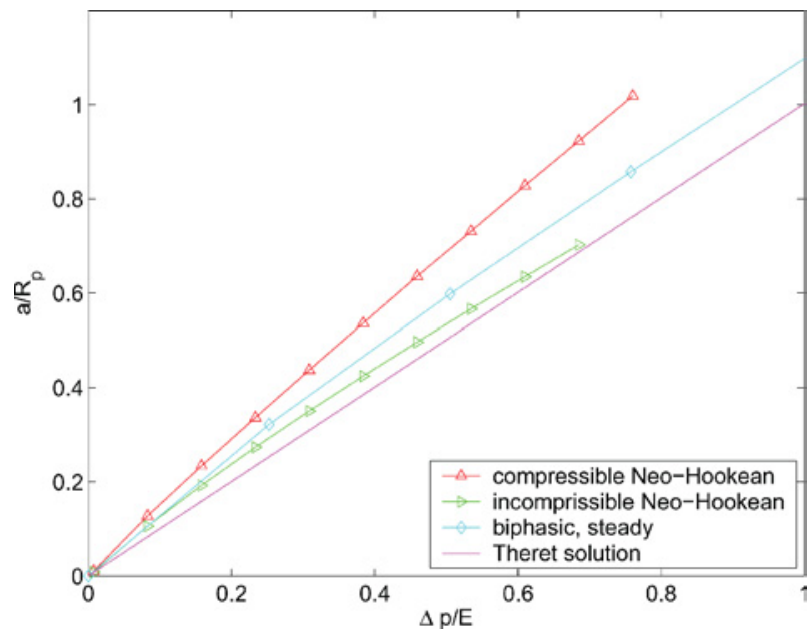

FIGURE 5. Normalized aspiration length $a / \boldsymbol{R}_{\mathrm{p}}$ as a function of the normalized pressure difference $\Delta p / E$, using a compressible and incompressible Neo-Hookean model, a compressible elastic biphasic model, and the Theret approximation, respectively.

model, the ratio of the initial aspiration length and the final steady state aspiration length is directly related to the ratio of the modulus of the elastic model, $G$ in Eq. (2), and the viscoelastic model, $G_{v}$ in Eq. (9). For this purpose the parameter $\beta$ is introduced, defined by

$$
G_{\mathrm{v}}=\beta G
$$

The definition of the Young's modulus $E$ is related to the shear modulus $G$ of the elastic model. At sufficiently small $t / \lambda$, the viscoelastic model Eq. (9) yields a (hypo)elastic

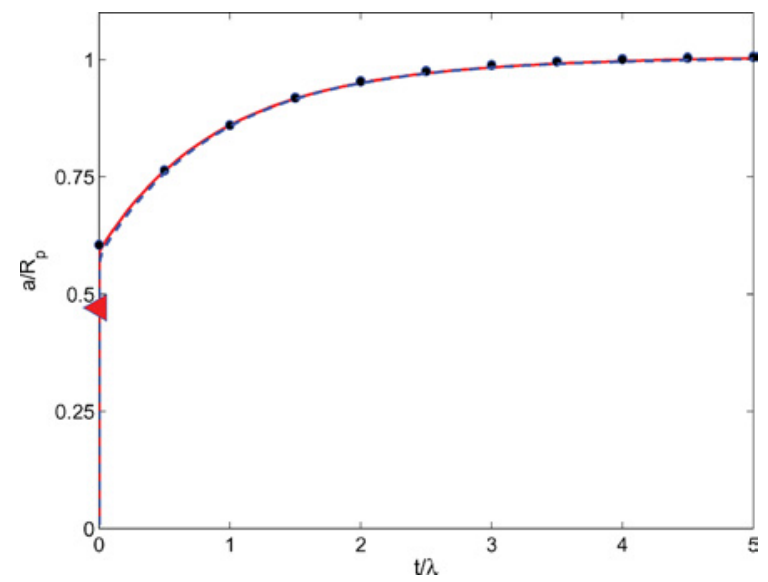

FIGURE 6. Normalized aspiration length $a / R_{\mathrm{p}}$ as a function of the normalized time $t / \lambda$. The solid dots indicate the experimental behavior of an average chondrocyte according to the parameters of Table. The applied pressure equals $362 \mathrm{~Pa}$. The solid line corresponds to the two-mode viscoelastic model, with $\beta=1.18, \lambda=15 \mathrm{~s}$, and $v=0.4$. The dashed line corresponds to the biphasic, two-mode viscoelastic model, with $\beta=1.2, \lambda=15 \mathrm{~s}$, and $v=0.4$. For the latter model the triangle marks the end of the pressure application, and the start of the creep response. 


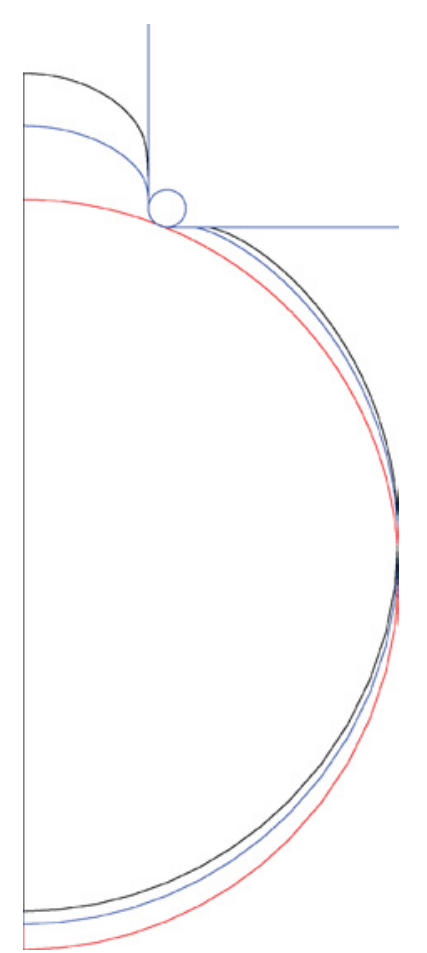

FIGURE 7. Shape of chondrocyte at three progressive aspiration stages: the initial shape, immediately after full application of the aspiration pressure, and the final steady shape. All using the two-mode compressible viscoelastic model.

response. Therefore, the effective stiffness for $t / \lambda \rightarrow 0$ equals $G+G_{\mathrm{v}}=(1+\beta) G$. If a steady state deformation is reached at sufficiently large $t / \lambda$, stresses associated with the viscoelastic mode relax completely, and the final response of the cell corresponds to an elastic response using the compressible neo-Hookean model with modulus $G$. Consequently, the elastic modulus $G$ can be estimated from the steady state aspiration length, while $\beta$ can subsequently be obtained from the initial aspiration length. The relaxation time $\lambda$ can subsequently be estimated from the time dependent response of the chondrocyte. Assuming compressible behavior at $v=0.4$, the two-mode viscoelastic model accurately predicts the average experimental response of chondrocytes, see Fig. 6 . The shape of the chondrocyte is depicted in Fig. 7 at $t=0$, at the end of the pressure application interval, and at the final steady configuration, respectively.

Previous studies have suggested that the compressible response of chondrocytes is due to a biphasic phenomenon, that is, due to diffusive drag between the fluid and solid phases. In the biphasic theory, the permeability parameter $k$ implicitly defines a time scale. Using the compressible neo-Hookean model in the biphasic equations (11) and (12), and the estimated values of the Poisson's ratio $(v=0.4)$ and the permeability parameter $k=7.7 \times 10^{-15} \mathrm{~m}^{4} /(\mathrm{N} \cdot \mathrm{s})$, it is not possible to reproduce fully the experimental response of the chondrocytes. However, using a two-mode viscoelas- tic model, the response is as depicted with the dashed line in Fig. 6 , at $\beta=1.2$ and $\lambda=15$ s. In this case the optimal value for $\beta$ was found iteratively, by systematic variation of $\beta$.

\section{DISCUSSION}

The objectives of this study were to investigate the ability of various constitutive models to predict the aspiration response of chondrocytes, and to examine the influence of the relative geometry of the cell and micropipette in determining parameters. Our findings suggest that a purely biphasic model cannot fully capture the experimentally observed creep response of chondrocytes, while a viscoelastic or biphasic viscoelastic model more accurately predicted cell response. These findings suggest that biphasic phenomena are likely not the primary mechanism responsible for cell viscoelasticity, although it may be responsible for observed changes in chondrocyte volume under mechanical stress. $^{11}$

The aspiration length of chondrocytes has been shown to be time dependent. ${ }^{11,22,23}$ After an initial elastic response that is proportional to the aspiration pressure, a creep response is observed before the aspiration length and the cell shape, reach a steady state. Both the initial response and the final steady state may be described using an elastic constitutive model. For this purpose the compressible neo-Hookean model was used. The use of the compressible model, in conjunction with its incompressible version, was motivated by the experimental results of ${ }^{11}$ that showed that chondrocytes are compressible and decrease their volume under mechanical compression. Given the intrinsic incompressibility of cells under hydrostatic pressure, ${ }^{24}$ this compressibility is likely due to exudation of fluid from the cell under a pressure gradient and indicative of a biphasic nature of chondrocytes. It therefore was hypothesized that the creep response was due to both the biphasic properties of the cell as well as an intrinsic viscoelastic response.

An assumption had to be made about the shape of the corner of the micropipette for the finite element simulations. Scanning electron microscopy images of the micropipette end showed a small curvature at the edge, of approximately $0.1 R_{\mathrm{p}}$, where $R_{\mathrm{p}}$ is the micropipette inner radius. In the micropipette simulations a circular shape was chosen with a slightly larger curvature: $0.15 R_{\mathrm{p}}$, to avoid the need for excessive mesh refinement near the contact area. The circular shape was advantageous for the numerical solution as opposed to a sharp corner because there were no discontinuities in the slope of the micropipette surface. Choosing a different shape or radius of the corner may alter the results, but Haider and Guilak, ${ }^{8}$ using a boundary element analysis, showed that changing this curvature did not alter the curve to as great a degree as the varying diameter ratios $\left(D_{\mathrm{c}} / D_{\mathrm{p}}\right)$. Despite the lack of singularities with the circular corner, all finite element simulations eventually ceased to converge, 
which, at least in part, is attributed to excessive element distortion near the circular edge of the pipette.

Theret et al. ${ }^{20}$ have derived an analytical expression of aspiration length assuming a half-space configuration, and an incompressible infinitesimal strain approximation. In that case the normalized aspiration length $a / R_{\mathrm{p}}$ is proportional to the normalized pressure $p / E$. The half space approximation implies that the finite size of the cell with respect to the micropipette diameter is not accounted for. It therefore is expected that for small ratios of the cell diameter to micropipette diameter this solution is inaccurate. This is indeed the case. If the $D_{\mathrm{c}} / D_{\mathrm{p}}$ ratio exceeds 3 , the Theret solution is indeed inaccurate, as demonstrated in Fig. 3.

The data presented in Fig. 4 and Table 1 allows one to calculate the Young's modulus of the cell at a given Poisson's ratio. For example, based on Table 1 the slope of the normalized aspiration length versus normal pressure curve equals 1.33 at $v=0.4$. Using the experimental results of $^{22}$ it can be concluded that the Young's modulus of nonosteoarthritic cells corresponding to the steady state during micropipette aspiration equals $0.48 \pm 0.22 \mathrm{kPa}$.

The trends observed in Fig. 4 are similar to those by Haider and Guilak ${ }^{7}$ in that the curves are slightly convex. In this study however, all values are above the analytical solution, while Haider and Guilak show values crossing from above to below one as the length increases. Two important differences between the two studies are the use of infinitesimal strain theory and the assumption of incompressibility in the previous study by Haider and Guilak, while the current study extends the constitutive model to account for large deformations and compressibility.

An important finding of this study was that the biphasic elastic model alone was not able to describe the time dependent creep behavior of chondrocytes during micropipette aspiration. This point was also demonstrated by the steady state results depicted in Fig. 5. If the pressure application is fast compared to the intrinsic time scale associated with the biphasic theory, the initial biphasic response approaches the incompressible solution, corresponding to the lowest curve in Fig. 5. Subsequently, the fluid is partially expelled from the chondrocyte, and compressibility effects enter the solution. This phenomenon explains why the biphasic solution predicts a deformation response that falls between the incompressible and the compressible solution. This finding also implies that the additional, time dependent aspiration length is at most equivalent to the difference in displacement between the fully incompressible and fully compressible curves (Fig. 5). However, the observed aspiration length from the initial state to the final steady state is much larger experimentally $^{22}$ than that predicted theoretically (Fig. 5). The limited additional creep like aspiration length is also observed in Fig. 6, where the triangle is the end of the pressure application phase. The intrinsic biphasic response manifests itself on a relatively short time scale, which is on the order of seconds (Fig. 6), and the aspiration length quickly increases to approach the experimental curve. After this, the viscoelastic creep response sets in.

In conclusion, the biphasic theory may indeed be used to predict the volumetric response of chondrocytes, but by itself does not explain the transient creep response during micropipette aspiration. The use of a compressible two-mode viscoelastic model alone is sufficient to capture the time dependent behavior of chondrocytes during micropipette experiments. Relative to the incompressible, infinitesimal strain half-space solution, the curvature of the cell significantly affects the results when the diameter ratio $D_{\mathrm{c}} / D_{\mathrm{p}}$ exceeds 3 , the compressibility of the cell substantially alters the estimation of the modulus of chondrocytes. Taken together with previous studies of the volumetric response of the chondrocyte to mechanical and osmotic stress, these findings suggest that the chondrocyte behaves as an intrinsically viscoelastic and "triphasic" material, whose volumetric response depends on both the mechanical and physicochemical environment around the cell. ${ }^{3,4,10,11,14}$

\section{ACKNOWLEDGMENTS}

This study was supported by grants from The Eindhoven University of Technology, the National Science Foundation \#DGE-9616283, and the National Institutes of Health \#AR50245, \#AG15768, AR48182.

\section{REFERENCES}

${ }^{1}$ Baaijens, F. An U-ALE formulation of 3-D unsteady viscoelastic flow. Int. J. Numer. Methods Eng. 36:1115-1143, 1993.

${ }^{2}$ Bachrach, N., W. Valhmu, E. Stazzone, A. Ratcliffe, W. Lai, and V. Mow. Changes in proteoglycan synthesis of chondrocytes in articular cartilage are associated with the time-dependent changes in their mechanical environment. J. Biomech. 28:15611570, 1995.

${ }^{3}$ Guilak, F. Compression-induced changes in the shape and volume of the chondrocyte nucleus. J. Biomech. 28:1529-1542, 1995.

${ }^{4}$ Guilak, F., G. Erickson, and H. Ting-Beall. The effects of osmotic stress on the viscoelastic and physical properties of articular chondrocytes. Biophys. J. 82:720-727, 2002.

${ }^{5}$ Guilak, F., and V. Mow. The mechanical environment of the chondrocyte: A biphasic finite element model of cell-matrix interactions in articular cartilage. J. Biomech. 33:1663-1673, 2000.

${ }^{6}$ Guilak, F., R. L. Sah, and L. A. Setton. Physical Regulation of Cartilage Metabolism, In: Mow VC, Hayes W. C., Editors. Basic Orthopaedic Biomechanics. 2nd ed. Philadelphia: LippincottRaven; 1997, p. 179-207.

${ }^{7}$ Haider, M., and F. Guilak. An axisymmetric boundary integral model for incompressible linear viscoelasticity: Application to the micropipette aspiration contact problem. J. Biomech. Eng. 122:236-244, 2000.

${ }^{8}$ Haider, M., and F. Guilak. An axisymmetric boundary integral model for assessing elastic cell properties in the micropipette aspiration contact problem. J. Biomech. Eng. 124:586-595, 2002.

${ }^{9}$ Hochmuth, R. Micropipette aspiration of living cells. J. Biomech. 33:15-22, 2000. 
${ }^{10}$ Hung, C., K. Costa, and X. Guo. Apparent and transient mechanical properties of chondrocytes during osmotic loading using triphasic theory and afm indentation. ASME Bioeng. Conf. BED50:625-626, 2001.

${ }^{11}$ Jones, W., H. Ting-Beall, G. Lee, S. Kelley, R. Hochmuth, and F. Guilak. Alterations in the young's modulus and volumetric properties of chondrocytes isolated from normal and osteoarthritic human cartilage. J. Biomech. 32:119-127, 1999.

${ }^{12}$ Koay, E., A. Shien, and K. Athanasiou. Creep indentation of single cells. J. Biomech. Eng. 125(3):334-341, 2003.

${ }^{13}$ Mow, V., S. Kuei, W. Lai, and C. Armstrong. Biphasic creep and stress relaxation of articular cartilage in compression: Theory and experiments. J. Biomech. Eng. 102:73-84, 1980.

${ }^{14}$ Mow, V., D. Sun, X. Guo, C. Hung, and W. Lai. Chondrocyteextracellular matrix interactions during osmotic swelling. ASME Bioeng. Conf. BED42:133-134, 1999.

${ }^{15}$ Poole, R. Imbalances of Anabolism and Catabolism of Cartilage Matrix Components in Osteoarthritis. In: Keuttner, K. E., Goldberg, V. M., eds. Osteoarthritic Disorders. AAOS Press, Rosemont, Illinois, USA, 1995, p. 247-260.

${ }^{16}$ Sato, M., D. Theret, L. Wheeler, N. Ohshima, and R. Nerem. Application of the micropipette technique to the measurement of cultured porcine aortic endothelial cell viscoelastic properties. J. Biomech. Eng. 112:263-268, 1990.

${ }^{17}$ Sengers, B., C. Oomens, and F. Baaijens. An integrated finite element approach to mechanics, transport and biosynthesis in tissue engineering. J. Biomech. Eng. 126:82-91, 2004.
${ }^{18}$ Setton, L., W. Zhu, and V. Mow. The biphasic poroviscoelastic behavior of articular cartilage: Role of the surface zone in governing the compressive behavior. J. Biomech. 26:581-592, 1993.

${ }^{19}$ Shin, D., and K. Athanasiou. Cytoindentation for obtaining cell biomechanical properties. J. Orthop. Res. 17:880-890, 1999.

${ }^{20}$ Theret, D., M. Levesque, M. Sato, R. Nerem, and L. Wheeler. The application of a homogeneous half-space model in the analysis of endothelial cell micropipette measurements. J. Biomech. Eng. 110:190-199, 1988.

${ }^{21}$ Trickey, W., F. Baaijens, T. Laursen, L. Alexopoulos, and F. Guilak. Determination of the Poisson's ratio of the cell: Recovery properties of chondrocytes after release from complete micropipette aspiration. J. Biomech. (Submitted), 2004a.

${ }^{22}$ Trickey, W., G. Lee, and F. Guilak. Viscoelastic properties of chondrocytes from normal and osteoarthritic human cartilage. J. Orthop. Res. 18:891-898, 2000.

${ }^{23}$ Trickey, W., T. Vail, and F. Guilak. The role of the cytoskeleton in the viscoelastic properties of human articular chondrocytes. J. Orthop. Res. 22:131-139, 2004b.

${ }^{24}$ Wilkes, R., and K. Athanasiou. The intrinsic incompressibility of osteoblast-like cells. Tissue Eng. 2:167-181, 1996.

${ }^{25} \mathrm{Wu}, \mathrm{J}$., W. Herzog, and M. Epstein. Modelling of location- and time-dependent deformation of chondrocytes during cartilage loading. J. Biomech. 32:563-572, 1999. 